$\mathrm{IC} / 96 / 43$

\title{
Two-loop renormalization group analysis of supersymmetric SO(10) models with an intermediate scale
}

\author{
Mar Bastero-Gil[ $]^{a}$ and Biswajoy Brahmachari[ $]^{b}$ \\ (a) Scuola Internazionale Superiore di Studi Avanzati \\ 34013 Trieste, ITALY. \\ (b) International Centre For Theoretical Physics, \\ 34100 Trieste, ITALY.
}

\begin{abstract}
$\underline{\text { Abstract }}$
Two-loop evolutions of the gauge couplings in a class of intermediate scale supersymmetric $\mathrm{SO}(10)$ models including the effect of third generation Yukawa couplings are studied. The unification scale, the intermediate scale and the value of the unification gauge coupling in these models are calculated and the gauge boson mediated proton decay rates are estimated. In some cases the predicted proton lifetime turns out to be in the border-line of experimental limit. The predictions of the top quark mass, the mass ratio $m_{b}\left(m_{b}\right) / m_{\tau}\left(m_{\tau}\right)$ from the two-loop evolution of Yukawa couplings and the mass of the left handed neutrino via see-saw mechanism are summarized. The lower bounds on the ratio of the VEVs of the two low energy doublets $(\tan \beta)$ from the requirement of the perturbative unitarity of the top quark Yukawa coupling up to the grand unification scale are also presented. All the predictions have been compared with those of the one-step unified theory.
\end{abstract}

Keywords: SO(10), SUSY, intermediate scale, Yukawa couplings.

\footnotetext{
${ }^{1}$ E-mail: bastero@stardust.sissa.it

${ }^{2}$ Address after 1st Oct 96: Department of Physics and Astronomy, University of Maryland, College Park, MD20742, USA. E-mail: biswajoy@ictp.trieste.it
} 


\section{Introduction and summary}

There is a general understanding in the literature that the LEP measurements of the gauge couplings at the scale $M_{Z}$ and a remarkable convergence of the couplings at the scale around $10^{16} \mathrm{GeV}$ is a hint to a one step supersymmetric unified theory. There are however several physical arguments suggesting that in a larger unified theory like $\mathrm{SO}(10)$ there may be an intermediate scale [1] corresponding to a left-right gauge symmetry breaking [2] somewhere around $10^{11}$ to $10^{12} \mathrm{GeV}$ based on neutrino physics [3, [4 as well as strong CP problem [5]. However, such a scale must not affect the gauge coupling unification constraints. Recently a number of studies have been performed to examine this question [6, [7, 8, 9, [1], [1]. In this paper we present a systematic two-loop analysis of a class of intermediate-scale supersymmetric $\mathrm{SO}(10)$ models varying the value of the strong coupling constant $\alpha_{3}\left(m_{Z}\right)$ in the experimentally allowed range. To begin with we calculate the predictions of (i) unification scale $M_{X}$, (ii) intermediate scale of B-L symmetry breaking $M_{I}$ (iii) the unification coupling $\alpha_{G}\left(M_{X}\right)$ and (iv) the proton lifetime in a two-loop renormalization group study.

The large value of the top quark mass measured by the CDF and DO collaborations at FERMILAB [12] is suggestive of a large top Yukawa coupling. Keeping this in mind we include the effect of all the third generation Yukawa couplings in the running of the gauge couplings. We note that in the large $\tan \beta$ region the effect of the bottom quark and the tau lepton Yukawa couplings also affect the gauge coupling evolution non-trivially, and one-step unification prefers a lower value of $\alpha_{3}\left(M_{Z}\right)=0.120$ instead of 0.124 obtained in the low $\tan \beta$ region. This effect also exists in the intermediate scale models. Later in this paper we focus our attention to the masses of fermions. 
We calculate the $(\mathrm{v})$ pole mass of the top quark when the top quark Yukawa coupling is at the quasi-infrared fixed point at the scale $M_{X}$. The predictions of (vi) the mass ratio $m_{b}\left(m_{b}\right) / m_{\tau}\left(m_{\tau}\right)$ also emerges from our analysis. The $\alpha_{3}$ dependence of these predictions are displayed graphically and they are mostly consistent with the observed values.

The ratio of VEVs $\tan \beta \equiv\left\langle H^{u}\right\rangle /\left\langle H^{d}\right\rangle$ is an unknown parameter of the supersymmetric models. However it has been noted that for very small values of $\tan \beta$ the top quark Yukawa coupling becomes very large before the scale $M_{X}$ and consequently it is possible to give a lower bound on $\tan \beta$ requiring the perturbative unitarity of the top Yukawa coupling up to the scale $M_{X}$. In this paper we make an analysis on the (vii) lower bound on $\tan \beta$ in such intermediate scale models. Taking the top quark Yukawa coupling to be arbitrarily large at the scale $M_{X}$ we derive an upper bound on it at the top scale $\left[h_{t}\left(m_{t}\right)\right]$ and then we convert this upper bound to a lower bound on the unknown parameter $\tan \beta$ for given values of the top quark mass.

We know that is difficult to understand the origin of a small neutrino-mass in the minimal supersymmetric standard model (MSSM) or in the minimal SU(5) GUT. In the presence of a right handed singlet neutrino the VEV of the $S U(2)_{L}$ doublet Higgs scalar will provide a Dirac mass to the neutrino which comparable to the masses of the other fermions. On the other hand we also know that in an intermediate scale model such as $\mathrm{SO}(10) \mathrm{GUT}$, it is possible to relate the mass of the left-handed neutrino to the inverse of the large lepton number violating Majorana mass of the right-handed neutrino $\left(M_{N} \sim M_{I}\right)$ by a see-saw mechanism [4]. We study the (viii) mass of the left-handed neutrino (of the third generation) in these models and calculate predictions for the tau neutrino mass by inputing of the values of the intermediate scales and various Yukawa couplings 
calculated from the first part of the paper. All our results can be compared with the one-step unification scenario in the limit $M_{I}=M_{X}$.

We will consider the value of $\alpha_{3}\left(M_{Z}\right)$ [13] in the range 0.110 to 0.130 , as the LEP measurements give $\alpha_{3}\left(M_{Z}\right)=0.124 \pm 0.006$ [14], which is typically 2 to 3 standard deviations larger than the value coming from $J / \Psi$-decay which is $\alpha_{3}\left(M_{Z}\right)=0.108 \pm 0.010$ [15], from lattice calculations which gives the value $\alpha_{3}\left(M_{Z}\right)=0.110 \pm 0.006$ [16], or from deep inelastic scattering experiments [17] which give $\alpha_{3}\left(M_{Z}\right)=0.112 \pm 0.002 \pm 0.004$.

This paper is organized as follows. In section (II) we motivate our models by a brief summary of an one-loop analysis. In section (III) we give the two-loop analysis of the mass scales. In section (IV) we give the predictions of top and the bottom quark masses and derive lower bound on $\tan \beta$. In section $(\mathrm{V})$ we discuss the predictions of tau neutrino mass from see-saw mechanism and in section (VI) we conclude. The relevant formulae have been summarized in the appendix.

\section{Models and scalar contents: A case study}

Before doing the rigorous analysis it is helpful to do a one-loop case-study and fix the scalar contents of various models to be considered afterwards. It is important to note for our purpose that an unified model with single intermediate scale is as predictive as a model with grand desert in the following sense. Three low energy observables in the gauge sector namely $\alpha_{1}\left(M_{Z}\right), \alpha_{2}\left(M_{Z}\right)$ and $\alpha_{3}\left(M_{Z}\right)$ can determine the three unknown parameters namely $M_{X}, M_{I}$ and $\alpha_{G}\left(M_{X}\right)$. Given $\alpha_{1}\left(M_{Z}\right)$ and $\alpha_{2}\left(M_{Z}\right)$ sufficiently precisely, there exists a unique value of $\alpha_{3}\left(M_{Z}\right)$ in the experimentally allowed region for 
which one gets $M_{X}=M_{I}$. Indeed in a model with more general scalar and fermion content one gets $M_{X} \neq M_{I}$, and thus, here we seek the models in which $M_{I} \leq M_{X}$.

In one loop approximation this unique value, for which $M_{X}=M_{I}$, is $\alpha_{3}\left(M_{Z}\right) \sim 0.1144$. It is noted by Lee and Mohapatra [8] that it is possible to get an intermediate scale $M_{I}<M_{X}$ in a number of models if $\alpha_{3} \neq 0.1144$. In this paper one of our objectives is to find this unique value of $\alpha_{3}$ in two-loop approximation and derive the ranges for the intermediate scale for the allowed range of $\alpha_{3}\left(M_{Z}\right)$.

Now let us write down the 1-loop renormalization group equation (RGE) of the three couplings introducing a general intermediate scale $M_{I}$ between $M_{Z}$ and $M_{X}$. We have used $b_{i}$ to denote the beta function coefficients below the intermediate scale and $b_{i}^{\prime}$ to denote them above the intermediate scale. The relations are,

$$
\alpha_{i}^{-1}\left(M_{Z}\right)=\alpha_{G}^{-1}\left(M_{X}\right)+\frac{b_{i}}{2 \pi} \ln \frac{M_{I}}{M_{Z}}+\frac{b_{i}^{\prime}}{2 \pi} \frac{M_{X}}{M_{I}} .
$$

A combination $\delta$ can be written [18] in which $b_{i}$ get eliminated but $b_{i}^{\prime}$ survive, as,

$$
\delta=7 \alpha_{3}^{-1}\left(M_{Z}\right)-12 \alpha_{2}^{-1}\left(M_{Z}\right)+5 \alpha_{1}^{-1}\left(M_{Z}\right) .
$$

Eqn.(11) and Eqn.(22) together leads to,

$$
\delta=\frac{1}{2 \pi}\left(7 b_{3}^{\prime}-12 b_{2}^{\prime}+5 b_{1}^{\prime}\right) \ln \frac{M_{X}}{M_{I}} \equiv \frac{\Delta}{2 \pi} \ln \frac{M_{X}}{M_{I}} .
$$

Now, if $\alpha_{1}\left(M_{Z}\right)=0.01696, \alpha_{2}\left(M_{Z}\right)=0.3371$ to get one-step unification in one loop we need $\alpha_{3}\left(M_{Z}\right)=0.1144$ which gives $\delta=0$ from Eqn.(2). However, experimentally $\alpha_{3}\left(M_{Z}\right)$ can be in the range 0.11 to 0.13 . Hence we obtain an allowed range in $\Delta$ which is crucial to get an intermediate 
scale,

$$
\delta^{\min } \leq \frac{\Delta}{2 \pi} \ln \frac{M_{X}}{M_{I}} \leq \delta^{\max }
$$

It may be noticed that when $\Delta$ is small we can achieve a large value for $\ln \frac{M_{X}}{M_{I}}$ leading to an intermediate scale well-below the unification scale. We will return to the inequality 1 in a moment. Now let us consider the symmetry breaking pattern,

$$
\begin{aligned}
S O(10) & \stackrel{M_{X}}{\longrightarrow} S U(3)_{c} \times S U(2)_{L} \times S U(2)_{R} \times U(1)_{(B-L)} \\
& \stackrel{M_{I}}{\longrightarrow} S U(3)_{c} \times S U(2)_{L} \times U(1)_{Y} \\
& \stackrel{M_{Z}}{\longrightarrow} S U(3)_{c} \times U(1)_{e m} .
\end{aligned}
$$

We restrict the type of Higgs representations above the intermediate scale $M_{I}$ by requiring that the supersymmetric SO(10) GUT emerges from an underlying superstring theory [19]. Restricting ourselves to only those Higgs scalars which can arise from simple superstring models with KacMoody levels one or two, we can have a restricted number of solutions of Eqn.(四). The various models can be characterized by a set of five integers $\left(n_{L}, n_{R}, n_{H}, n_{C}, n_{d}\right)$, where $n_{C}$ refers to the number of $(8,1,1,0), n_{H}$ means the number of $(1,2,2,0)$ fields and $n_{L}$ and $n_{R}$ means the number of $(1,2,1,1)+(1,2,1,-1)$ and $(1,1,2,1)+(1,1,2,-1)$ fields under the intermediate symmetry gauge group and $n_{d}$ refers to the number of pairs of Higgs doublets below the scale $M_{I}$. To further restrict the number of models we have the following assumptions.

- The theory below the intermediate scale in strictly the Minimal Supersymmetric Standard Model (MSSM). We will also consider an example where the theory below $M_{I}$ has four Higgs doublets

\footnotetext{
3 In this case the combination in the Eqn. (2) is no longer orthogonal to the beta function coefficients be-
} 
instead of two in the standard model.

- It should be possible to get an intermediate scale which is at least one to two orders of magnitude below the unification scale $\left(\ln \frac{M_{X}}{M_{I}}=2.3-4.6\right)$.

Using the beta function coefficients listed in the appendix Eqn. (州 gives,

$$
-10 \leq\left(-9+21 n_{c}-9 n_{H}+6 n_{R}-9 n_{L}\right) \leq 6 .
$$

The models satisfying the above inequality are tabulated in Table 1 which are numbered in the decreasing order of minimality. We have included one scenario namely model VIII which has a color octet above the scale $M_{I}$. This scenario has $\Delta=0$ and thus it can accommodate a large splitting between $M_{I}$ and $M_{X}$. In scenario IX the theory below the scale $M_{I}$ has four Higgs doublets $\left(n_{d}=2\right)$.

\begin{tabular}{|c||ccccc||c|}
\hline Model & $n_{L}$ & $n_{R}$ & $n_{H}$ & $n_{c}$ & $n_{d}$ & $\Delta$ \\
\hline I & 0 & 2 & 1 & 0 & 1 & -6 \\
II & 0 & 3 & 1 & 0 & 1 & 0 \\
III & 0 & 4 & 1 & 0 & 1 & 6 \\
IV & 0 & 3 & 2 & 0 & 1 & -9 \\
V & 0 & 4 & 2 & 0 & 1 & -3 \\
VI & 0 & 5 & 2 & 0 & 1 & 3 \\
VII & 1 & 5 & 2 & 0 & 1 & -6 \\
\hline VIII & 1 & 1 & 1 & 1 & 1 & 0 \\
\hline IX & 0 & 3 & 2 & 1 & 2 & - \\
\hline
\end{tabular}

Table 1: The minimal models which satisfy the condition in Eqn.(5). When the quantity $\Delta$ is positive (negative) the model gives rise to an intermediate scale for the lower (higher) values of $\alpha_{3}\left(M_{Z}\right)$ than the one step unification case for which $\alpha_{3}\left(M_{Z}\right)=0.1144$ at the one-loop level. In the case $\Delta=0$ the intermediate scale is unconstrained at the one-loop level.

low $M_{I}$. However it is straight-forward to derive an equivalent combination for this case. Interesting low-energy phenomenology of such a model can be found in Ref. [20]. 


\section{Two loop analysis of mass scales, unified coupling and proton decay}

In the two-loop case the gauge couplings evolve according to the equation,

$$
\frac{d \alpha_{i}}{d t}=\frac{b_{i}}{2 \pi} \alpha_{i}^{2}+\sum_{j} \frac{b_{i j}}{8 \pi^{2}} \alpha_{i}^{2} \alpha_{j}-\sum_{k} \frac{a_{i k}}{8 \pi^{2}} \alpha_{i}^{2} Y_{k},
$$

where the Yukawa couplings can be defined by the superpotential invariant under the intermediate symmetry, as,

$$
\mathcal{W}_{\mathcal{Y}}=h_{Q_{1}} Q^{T} \tau_{2} \phi_{1} Q^{c}+h_{Q_{2}} Q^{T} \tau_{2} \phi_{2} Q^{c}+h_{L_{1}} L^{T} \tau_{2} \phi_{1} L^{c}+h_{L_{2}} L^{T} \tau_{2} \phi_{2} L^{c}+\text { h.c. }
$$

where we have denoted the quarks and leptons by the obvious notation $Q, Q^{c}$ and $L, L^{c}$. At the GUT scale, we have $h_{Q_{1}}\left(M_{X}\right)=h_{L_{1}}\left(M_{X}\right)=h_{1}\left(M_{X}\right)$ and $h_{Q_{2}}\left(M_{X}\right)=h_{L_{2}}\left(M_{X}\right)=h_{2}\left(M_{X}\right) . \phi_{1,2}$ are two Higgs bidoublets embedded in $10_{1,2}$ scalars of $\mathrm{SO}(10)$. We have used the notation,

$$
t=\ln \mu \quad, \quad \alpha=\frac{g^{2}}{4 \pi}, \quad Y=\frac{h^{2}}{4 \pi},
$$

where $\mathrm{g}$ and $\mathrm{h}$ refers to the gauge and Yukawa couplings respectively. The coefficients are given in detail in the appendix. When $Y_{1}\left(M_{X}\right)$ and $Y_{2}\left(M_{X}\right)$ take large values of order $O(1)$ the Yukawa couplings affect the running of the gauge couplings most. In this case $Y_{t}$ and $Y_{b}$ have almost equal values at the low energy scale. Such a situation corresponds to large $\tan \beta$. On the other hand if $Y_{2}\left(M_{X}\right)<<Y_{1}\left(M_{X}\right)$ at the scale $M_{X}$ the top-bottom mass hierarchy is due to Yukawa coupling hierarchy and consequently $\tan \beta$ is order $\mathrm{O}(1)$. We will refer to this case as the small $\tan \beta$ scenario.

In models I, II, III and VIII $\tan \beta$ is always large.

First, we present the results of the unification scale. The variation of the unification scales with 
that of $\alpha_{3}\left(M_{Z}\right)$ have been plotted in Figure (1a). The predictions of the intermediate scale have been plotted in Figure (1b) and that of the unification coupling $\alpha_{G}\left(M_{X}\right)$ have been plotted in Figure (1c) for various models. The grand desert case can be recovered from the meeting point of all the curves in Figure (1b) that is when the intermediate scale is equal to the GUT scale. In the low $\tan \beta$ region the meeting occurs at the value $\alpha_{3}\left(M_{Z}\right)=0.124$ and in the high $\tan \beta$ region it occurs at the value $\alpha_{3}\left(M_{Z}\right)=0.120$. For all other values of $\alpha_{3}\left(M_{Z}\right)$ it is possible to have an intermediate scale. In model VI the unification scale becomes low in the low $\alpha_{3}$ region; for model $\mathrm{V}$ the same thing happens for high $\alpha_{3}\left(M_{Z}\right)$ region. As the dimension five proton decay can be suppressed in the $\mathrm{SO}(10)$ models by some additional mechanism [22], we plot the dominant dimension six decay mode in Figure (1d). The proton decay rate has been calculated using the formula below in which we have taken the mass of the heavy gauge bosons as $M_{X}$.

$$
\Gamma\left(p \longrightarrow e^{+} \gamma\right)=\frac{m_{N} \alpha^{2}}{64 \pi f_{\pi}^{2}}\left[\frac{4 \pi \alpha_{G} A_{R}}{M_{X}^{2}}\right]^{2}\left[1+\left(1+\left|V_{u d}\right|^{2}\right)^{2}\right][1+D+F]
$$

The values of parameters used in Eqn. (9), have been listed in the appendix. The variation of decay mode vis-a-vis the variation of $\alpha_{3}\left(M_{Z}\right)$ comes not only from the variation of $M_{X}$ and $\alpha_{G}$ but also due to a (mild) renormalization of the proton decay operator [24, 25] up to the scale of $1 \mathrm{GeV}$ which has been parameterized in the factor $A_{R}$. When $\alpha_{3}\left(M_{Z}\right)$ is above $0.130, \alpha_{3}(1 \mathrm{GeV})$ becomes very large and the perturbative calculation is not dependable. In our estimation of $A_{R}$ we have made taken into account the renormalization of the operator due to $S U(3)$ color and $S U(2)_{L}$ interactions only. This is a reasonable approximation as in most cases $M_{I}$ is large enough and the $S U(2)_{R}$ renormalization effects exist only beyond $M_{I}$. In the one-step unification case our value of

\footnotetext{
${ }^{4}$ For moderate values of $\tan \beta$ unification occurs for values of $\alpha_{3}\left(M_{Z}\right)$ within 0.120 and 0.124 . Low energy threshold corrections 21] assuming universality of gaugino masses will shift this whole range upwards.
} 
the parameter $A_{R}$ agrees with that of Ref. [25].

The lifetime of proton has been plotted in Figure (4). Model V predicts large proton decay rates (in the borderline of experimental limit) for the values of $\alpha_{3}\left(M_{Z}\right)$ beyond 0.123 . Similarly model VI predicts equally large rate of proton decay for lower values of $\alpha_{3}\left(M_{Z}\right)$ below 0.114 . The models except I, VIII and IX predicts a lower proton lifetime than the minimal one step unification scenario when there is an intermediate scale. This is encouraging from the forthcoming proton decay experiments where these models can be probed. In fact if the experiments at SuperKamiokande excludes the minimal unification scenario it will also exclude all the present models except model I, VIII and IX.

Before concluding this section let us make a small remark about the curvatures visible in the plots near the meeting point of the models. These curvatures are more pronounced in models $\mathrm{V}$ and VII. Let us consider Figure (1b) for example. Near the GUT scale the Yukawa couplings are large and they fall quickly below the GUT scale. The Yukawa couplings tend to pull the individual lines towards the low $\alpha_{3}$ region wheres in the high $\alpha_{3}$ models (like V and VII) the gauge interactions have exactly the reverse effect. This causes the curvature in the graphs near the GUT scale which is purely a two-loop effect.

\section{Fermion masses and Yukawa couplings}

The simultaneous evolution of gauge and Yukawa couplings enables us to calculate the following Dirac type masses of quarks and leptons, in respective scales denoted in the parenthesis, by the 
following relations,

$$
\begin{aligned}
m_{t}\left(m_{t}\right) & =\sqrt{4 \pi Y_{t}\left(m_{t}\right)} \frac{v}{\sqrt{2}} \sin \beta, \\
m_{b}\left(m_{b}\right) & =\sqrt{4 \pi Y_{b}\left(m_{t}\right)} \frac{v}{\sqrt{2}} \cos \beta \eta_{b}, \\
m_{\tau}\left(m_{\tau}\right) & =\sqrt{4 \pi Y_{\tau}\left(m_{t}\right)} \frac{v}{\sqrt{2}} \cos \beta \eta_{\tau} . \\
m_{\nu_{\tau}}\left(M_{I}\right) & =\sqrt{4 \pi Y_{L 1}\left(M_{I}\right)} \frac{v}{\sqrt{2}} \sin \beta .
\end{aligned}
$$

The factors $\eta_{b}$ and $\eta_{\tau}$ takes into account the running of the masses of $m_{b}$ and $m_{\tau}$ to their respective scales starting from $m_{t}$. We have used the formula of reference [26] to calculate $\eta_{b}$ and $\eta_{\tau}$ taking into account the three loop QCD effects and one loop QED effects. We have got $\eta_{\tau}=1.017$ assuming $\alpha_{e m}^{-1}\left(M_{Z}\right)=127.9$. The values of $\eta_{b}$ are plotted in the appendix. We start from a pair of values $Y_{1}\left(M_{X}\right)$ and $Y_{2}\left(M_{X}\right)$. Using the RGE for the Yukawa couplings given in the appendix we calculate the values of the couplings $Y_{t}\left(m_{t}\right), Y_{b}\left(m_{t}\right)$ and $Y_{\tau}\left(m_{t}\right)$. At the scale $M_{I}$ the boundary conditions for the Yukawa couplings are,

$$
\begin{aligned}
& Y_{Q 1}\left(M_{I}\right)=Y_{t}\left(M_{I}\right), Y_{Q 2}\left(M_{I}\right)=Y_{b}\left(M_{I}\right) \text { and } Y_{L 2}\left(M_{I}\right)=Y_{\tau}\left(M_{I}\right) \text { when } n_{H}=2, \\
& Y_{Q}\left(M_{I}\right)=Y_{t}\left(M_{I}\right)=Y_{b}\left(M_{I}\right), \text { and } Y_{L}\left(M_{I}\right)=Y_{\tau}\left(M_{I}\right) \text { when } n_{H}=1
\end{aligned}
$$

Using Equation Eqn. (12), which has the least experimental error we calculate the value of $\cos \beta$. Once the value of $\cos \beta$ is known the predictions for $m_{t}\left(m_{t}\right)$ and $m_{b}\left(m_{b}\right)$ follows from Eqn. (10) and Eqn. (11). The running mass of the top quark has been calculated by iterative procedure using the condition

$$
m_{t}\left(m_{t}\right)=m_{t} .
$$


The pole mass 23] has been calculated from the running mass in Eqn. (16) using the equation

$$
m_{t}^{\text {pole }}=m_{t}\left(m_{t}\right)\left[1+\frac{4 \alpha_{3}\left(m_{t}\right)}{3 \pi}\right]
$$

for each value of $\alpha_{3}\left(M_{Z}\right)$. The predictions of $m_{t}^{\text {pole }}$ is plotted in Figure (2a). The value of $R_{b}\left(m_{t}\right) \equiv$ $\frac{m_{b}\left(m_{t}\right)}{m_{\tau}\left(m_{t}\right)}$ are plotted in Figure $(2 \mathrm{c})$, and when the prediction of $m_{b}\left(m_{t}\right)$ is extrapolated to the mass scale of the bottom quark we get Figure $(2 \mathrm{~d})$ using $m_{\tau}\left(m_{\tau}\right)=1.777 \mathrm{GeV}$. A few comments are in order.

- When we decrease $\sin \beta(\tan \beta)$, the reduction in the bottom quark Yukawa effect increases the value of $h_{t}\left(m_{t}\right)$. This is visible in the plot $(2 \mathrm{a})$. However, once the value of $Y_{2}\left(M_{X}\right)$ is smaller than $10^{-4}$, the effect of the bottom quark Yukawa coupling on $Y_{t}$ virtually ceases to exist. At this stage a further reduction in $\sin \beta(\tan \beta)$ causes a large reduction in the predicted value of the top mass. And in fact for $Y_{2}\left(M_{X}\right)=10^{-5}$ the predicted top mass falls down to $161 \mathrm{GeV}$.

- The experimentally allowed range of $m_{b}\left(m_{b}\right)$ is between 4.10 and $4.50 \mathrm{GeV}$ [13]. However masses as high as $5.2 \mathrm{GeV}$ are also considered in the literature. The predictions put strong constraints on the model. Especially for the low $\alpha_{3}\left(M_{Z}\right)$ case, high $\tan \beta$ regime is disfavored. In the low $\tan \beta$ scenario the predictions shift towards higher $\alpha_{3}\left(M_{Z}\right)$ which leads to a bigger $\eta_{b}$ [see Fig.(7)] and hence a larger predicted value of $m_{b}\left(m_{b}\right)$.

- The lower bounds on $\tan \beta(\sin \beta)$ follows directly from an upper bound onf $Y_{t}\left(m_{t}\right)$. We calculate the bound by the following procedure. Taking the value of $Y_{t}\left(M_{X}\right)$ to be arbitrarily large we calculate the upper bounds for $Y_{t}\left(m_{t}\right)$ for various $Y_{2}\left(M_{X}\right)$. The absolute upper bound is converted

\footnotetext{
${ }^{5}$ It is well-known that the Yukawa evolution equations have an infrared quasi-fixed point structure [27].
} 
into a lower bound for $\tan \beta$ by Eqn. (10) and Eqn. (17). These bounds have been plotted in Fig. (2b).

\section{See-saw mechanism and neutrino mass}

If the neutrino is a Majorana particle it can have a lepton number violating Majorana mass term. $\mathrm{SO}(10)$ GUT has a natural mechanism to generate a large Majorana mass of the right handed neutrino. In this case the neutrino mass matrix has the form,

$$
\mathcal{M}=\left(\begin{array}{cc}
0 & m^{d} \\
m^{d} & M
\end{array}\right),
$$

where $m^{d}$ is a $3 \times 3$ Dirac mass matrix and $\mathrm{M}$ is a $3 \times 3$ Majorana mass matrix in general. When the matrix $\mathcal{M}$ is diagonalized we get two eigenvalues of orders $M$ and $\left[m^{d}\right]^{2} / M$. The latter eigenvalue can in principle explain the smallness of the mass of the left-handed neutrino when $\mathrm{M}$ is large.

In our models the intermediate $B-L$ symmetry is broken by the Higgs scalars $16+\overline{16}$ fields of $\mathrm{SO}(10)$. We will consider two different scenarios by which Majorana mass of the right handed neutrino can be generated.

(a) Using a higher dimensional operator of the form $\frac{h}{M_{X}} 16_{F} 16_{F} 16_{H} 16_{H}$ written in terms of $\mathrm{SO}(10)$ representations. The subscripts $\mathrm{F}$ and $\mathrm{H}$ mean fermions and scalars respectively. When $16_{H}$ gets a VEV a large Majorana mass of the order $h v_{R}^{2} / M_{X}$ is generated.

(b) Introduction of additional singlets to have a generalized see-saw mechanism [8, 28]. In this case the mass is effectively generated from a $3 \times 3$ see-saw matrix. This case is interesting as an effective 
$2 \times 2$ see-saw matrix can be recovered purely from renormalizable interactions when the singlet scalars get their VEVs.

The prediction of the mass of the left-handed neutrino in the case (a) is well-known. We recast it in terms of the parameters being evaluated by RGEs in our cases (we have used $M_{I}=g_{R} v_{R}$ ), as,

$$
h m_{\nu}\left(M_{I}\right)=\frac{\left[h_{L 1}\left(M_{I}\right) v^{u}\right]^{2}}{v_{R}^{2} / M_{X}}=8 \pi^{2} \alpha_{2 R}\left(M_{I}\right) \frac{Y_{L 1}\left(M_{I}\right)\left[v^{2} \tan ^{2} \beta /\left(1+\tan ^{2} \beta\right)\right]}{M_{I}^{2} / M_{X}} .
$$

In the case (b) 8 , 28], the $3 \times 3$ mass matrix in the basis $\left(\left(\nu_{a}, N_{a}, S_{a}\right)\right.$, where a=1,2,3 and $S_{a}$ are the singlets) is,

$$
\mathcal{M}=\left(\begin{array}{ccc}
0 & h_{1} v^{u} & 0 \\
h_{1} v^{u} & 0 & h^{\prime} v_{R} \\
0 & h^{\prime} v_{R} & M_{s}
\end{array}\right),
$$

where $M_{s}$ is the mass of the singletfo. In this case the small mass of the left-handed neutrino arises purely from the renormalizable interactions, as,

$$
h^{\prime} m_{\nu}\left(M_{I}\right)=\frac{\left[h_{L 1}\left(M_{I}\right) v^{u}\right]^{2}}{v_{R}^{2} / M_{s}}=8 \pi^{2} \alpha_{2 R}\left(M_{I}\right) \frac{Y_{L 1}\left(M_{I}\right)\left[v^{2} \tan ^{2} \beta /\left(1+\tan ^{2} \beta\right)\right]}{M_{I}^{2} / M_{s}} .
$$

In Eqn. (21) we will consider $M_{s}=M_{I}$. When $M_{s}=M_{X}$ we recover Eqn. (19) from Eqn. (21). In both the cases we will assume,

$$
v^{2}=\left(v^{u}\right)^{2}+\left(v^{d}\right)^{2}=247^{2} / 2 G e V^{2}
$$

In Figure (3a) and Figure (3b) we have plotted the left handed neutrino masses in scenarios (a) and (b) modulo the unknown Yukawa couplings $h$ and $h^{\prime}$ in various models as a function of $\alpha_{3}\left(M_{Z}\right)$. These numbers will get renormalized when they are extrapolated up to the scale of $1 \mathrm{GeV}$

\footnotetext{
${ }^{6}$ For the detailed superpotential see Ref 8
} 
[29, 30, 31]. In Ref [31 it has been noted that the Yukawa couplings affect the extrapolation of the see-saw formula and the tau neutrino mass increases. This extrapolation will change the mass of the left handed neutrino by a factor of the order of unity.

We know that a tau neutrino mass of the order of a few electron volts is preferable if neutrino is to be a candidate for the Hot Dark Matter (HDM). We see that in scenario (a) a tau neutrino in the range of 1-10 eV can be achieved in all the models depending on the value of $\alpha_{3}\left(M_{Z}\right)$. On the other hand, scenario (b) can predict a tau neutrino mass in the $1-10 \mathrm{eV}$ range for models $\mathrm{V}$, II and VI. However, model VI and V have potential problems with proton decay around the values of $\alpha_{3}\left(M_{Z}\right)$ needed to produce a correct order of the mass of the $\nu_{\tau}$, whereas model II predicts a lower value for the bottom quark mass.

\section{Conclusions}

We have considered a class of minimal models for which an intermediate scale in a $\mathrm{SO}(10)$ GUT can be achieved. We have found that a small number $\Delta$ can be defined, the sign of which can tell whether the unification occurs for a low value of $\alpha_{3}$ or a high value of $\alpha_{3}$ compared to the one-step unification. After listing the minimal models in which an intermediate scale can be generated, we have done a two-loop analysis of the gauge couplings to find the unification scale, the intermediate scale and the unification gauge coupling. These predictions are in general functions of the input $\alpha_{3}\left(M_{Z}\right)$. We have plotted the predictions in Figure 1. The predictions for one-step unification can be recovered from the meeting point of all the branches in these figures for which $M_{I}=M_{X}$. The 
gauge boson mediated proton decay rates have been calculated after renormalizing the proton decay operator to the scale of $1 \mathrm{GeV}$. These predictions are also plotted in the Figure 1. In some models the predicted proton life-time is in the borderline of experimental exclusion limits. These models can be tested in the SuperKamiokande experiments soon.

We have also done a parallel two-loop analysis of the Yukawa sector of these models. The combined running of the gauge and Yukawa couplings enable us to calculate the predictions of the top and bottom quark masses using the mass of the tau lepton $m_{\tau}\left(m_{\tau}\right)$ as an input. The predictions of the top mass is always in the range given by the CDF and D0 collaborations. The bottom quark mass is not always in the range $4.10-4.50 \mathrm{GeV}$ as quoted in the review of particle properties [13]. Thus predictions of the bottom quark mass is a good indicator by which one could compare these models relative to each other. Running of the Yukawa coupling also gives a lower bound on the parameter $\tan \beta$ from the requirement that the top quark Yukawa coupling remains perturbative up to the scale of unification.

The determination of the intermediate scale and the running of the Yukawa couplings enable us to calculate the predictions of the neutrino masses via see-saw mechanism. The Dirac type entry in the see-saw matrix can be fixed from the running of the neutrino Yukawa coupling. The predictions of the tau neutrino for various values of $\alpha_{3}$ have been plotted. A mass of the tau neutrino in the range 1-10 electron volts can be achieved in various models. It can be noted from the plot of the neutrino masses that in the one-step unification model the mass of the tau neutrino is in the range of $10^{-2}$ electron volts only, making it less attractive as a dark matter candidate. 
The scenario VIII can be a good candidate for string unification with the unification scale near the plank scale and the unification coupling larger than the one-step unification case.

\section{Acknowledgments}

We thank R. N. Mohapatra and G. Senjanović for useful comments. We also thank J. Kubo for sending us a numerical subroutine which we have used in our analysis.

\section{Appendix}

\subsection{Beta function coefficients for the Yukawa couplings}

It is easy to write down the evolution equations for the Yukawa couplings defined by $\sqrt{4 \pi Y_{i j k}} \phi_{i} \phi_{j} \phi_{k}$, as,

$$
\frac{d Y_{i j k}}{d t}=2 Y_{i j k}\left[\gamma_{i}+\gamma_{j}+\gamma_{k}\right]
$$

The scale $\mathrm{t}$ is defined as $t=\ln \mu$. In the two loop approximation we can write,

$$
\gamma_{i}=\gamma_{i}^{(1)}+\gamma_{i}^{(2)}
$$

The superscripts in parenthesis denote the order of perturbation theory. The anomalous dimensions for the superfields below the scale $M_{I}$ are given below. In the one-loop anomalous dimensions are,

$$
\begin{aligned}
\gamma_{L}^{(1)} & =\frac{1}{4 \pi}\left[Y_{\tau}-\frac{3}{2} \alpha_{2}-\frac{3}{10} \alpha_{1}\right] \\
\gamma_{\bar{E}}^{(1)} & =\frac{1}{4 \pi}\left[2 Y_{\tau}-\frac{6}{5} \alpha_{1}\right]
\end{aligned}
$$




$$
\begin{aligned}
\gamma_{\bar{D}}^{(1)} & =\frac{1}{4 \pi^{2}}\left[2 Y_{b}-\frac{8}{3} \alpha_{3}-\frac{4}{30} \alpha_{1}\right], \\
\gamma_{\bar{U}}^{(1)} & =\frac{1}{4 \pi}\left[2 Y_{t}-\frac{8}{3} \alpha_{3}-\frac{8}{15} \alpha_{1}\right], \\
\gamma_{Q}^{(1)} & =\frac{1}{4 \pi}\left[Y_{t}+Y_{b}-\frac{8}{3} \alpha_{3}-\frac{3}{2} \alpha_{2}-\frac{1}{30} \alpha_{1}\right], \\
\gamma_{H_{1}}^{(1)} & =\frac{1}{4 \pi}\left[Y_{\tau}+3 Y_{b}-\frac{3}{2} \alpha_{2}-\frac{3}{10} \alpha_{1}\right], \\
\gamma_{H_{2}}^{(1)} & =\frac{1}{4 \pi}\left[3 Y_{t}-\frac{3}{2} \alpha_{2}-\frac{3}{10} \alpha_{1}\right] .
\end{aligned}
$$

The two-loop anomalous dimensions are;

$$
\begin{aligned}
& \gamma_{L}^{(2)}= \frac{1}{16 \pi^{2}}\left[-Y_{\tau}\left(\gamma_{\bar{E}}^{(1)}+\gamma_{H_{1}}^{(1)}\right)\right. \\
&\left.-\left(\frac{3}{2} \alpha_{2}+\frac{3}{10} \alpha_{1}\right) \gamma_{L}^{(1)}+\frac{3}{2} b_{2} \alpha_{2}^{2}+\frac{3}{10} b_{1} \alpha_{1}^{2}\right] \\
& \gamma_{\bar{E}}^{(2)}= \frac{1}{16 \pi^{2}}\left[-2 Y_{\tau}\left(\gamma_{L}^{(1)}+\gamma_{H_{1}}^{(1)}\right)\right. \\
&\left.-\frac{6}{5} \alpha_{1} \gamma_{\bar{E}}^{(1)}+\frac{6}{5} b_{1} \alpha_{1}^{2}\right] \\
& \gamma_{\bar{D}}^{(2)}= \frac{1}{16 \pi^{2}}\left[-2 Y_{b}\left(\gamma_{Q}^{(1)}+\gamma_{H_{1}}^{(1)}\right)\right. \\
&\left.-\left(\frac{8}{3} \alpha_{3}+\frac{2}{15} \alpha_{1}\right) \gamma_{\bar{D}}^{(1)}+\frac{8}{3} b_{3} \alpha_{3}^{2}+\frac{2}{15} b_{1} \alpha_{1}^{2}\right] \\
& \gamma_{\bar{U}}^{(2)}= \frac{1}{16 \pi^{2}}\left[-2 Y_{t}\left(\gamma_{Q}^{(1)}+\gamma_{H_{2}}^{(1)}\right)\right. \\
&\left.-\left(\frac{8}{3} \alpha_{3}-\frac{8}{15} \alpha_{1}\right) \gamma_{\bar{U}}^{(1)}+\frac{8}{3} b_{3} \alpha_{3}^{2}+\frac{8}{15} b_{1} \alpha_{1}^{2}\right] \\
& \gamma_{H_{1}}^{(2)}= \frac{1}{16 \pi^{2}}\left[-Y_{\tau}\left(\gamma_{L}^{(1)}+\gamma_{\bar{E}}^{(1)}\right)-3 Y_{b}\left(\gamma_{Q}^{(1)}+\gamma_{\bar{D}}^{(1)}\right)\right. \\
& \gamma_{Q}^{(2)}= \frac{1}{4 \pi}\left[-Y_{t}\left(\gamma_{\bar{U}}^{(1)}+\gamma_{H_{2}}^{(1)}\right)-Y_{b}\left(\gamma_{\bar{D}}^{(1)}+\gamma_{H_{1}}^{(1)}\right)\right. \\
&\left.\left.\alpha_{3}+\frac{3}{2} \alpha_{2}+\frac{1}{30} b_{1} \alpha_{1}\right) \gamma_{Q}^{(1)}+\frac{8}{3} b_{3} \alpha_{3}^{2}+\frac{3}{2} b_{2} \alpha_{2}^{2}+\frac{1}{30} b_{1} \alpha_{1}^{2}\right] \\
&= \\
&=
\end{aligned}
$$




$$
\begin{aligned}
& \left.-\left(\frac{3}{2} \alpha_{2}+\frac{3}{10} \alpha_{1}\right) \gamma_{Q}^{(1)}+\frac{3}{2} b_{2} \alpha_{2}^{2}+\frac{3}{10} b_{1} \alpha_{1}^{2}\right] \\
\gamma_{H_{2}}^{(2)}= & \frac{1}{16 \pi^{2}}\left[-3 Y_{t}\left(\gamma_{Q}^{(1)}+\gamma_{\bar{U}}^{(1)}\right)\right. \\
& \left.-\left(\frac{3}{2} \alpha_{2}+\frac{3}{10} \alpha_{1}\right) \gamma_{H_{2}}^{(1)}+\frac{3}{2} b_{2} \alpha_{2}^{2}+\frac{3}{10} b_{1} \alpha_{1}^{2}\right] .
\end{aligned}
$$

Above the scale $M_{I}$ the one-loop anomalous dimensions are,

$$
\begin{aligned}
\gamma_{Q}^{(1)} & =\frac{1}{4 \pi}\left[2 Y_{Q 1}+2 Y_{Q 2}-\frac{8}{3} \alpha_{c}-\frac{3}{2} \alpha_{L}-\frac{1}{12} \alpha_{B-L}\right] \\
\gamma_{Q^{c}}^{(1)} & =\frac{1}{4 \pi}\left[2 Y_{Q 1}+2 Y_{Q 2}-\frac{8}{3} \alpha_{c}-\frac{3}{2} \alpha_{R}-\frac{1}{12} \alpha_{B-L}\right] \\
\gamma_{L}^{(1)} & =\frac{1}{4 \pi}\left[2 Y_{L 1}+2 Y_{L 2}-\frac{3}{2} \alpha_{L}-\frac{3}{4} \alpha_{B-L}\right], \\
\gamma_{L^{c}}^{(1)} & =\frac{1}{4 \pi}\left[2 Y_{L 1}+2 Y_{L 2}-\frac{3}{2} \alpha_{R}-\frac{3}{4} \alpha_{B-L}\right], \\
\gamma_{\phi_{i}}^{(1)} & =\frac{1}{4 \pi}\left[3 Y_{Q i}+Y_{L i}-\frac{3}{2} \alpha_{L}-\frac{3}{2} \alpha_{R}\right] .
\end{aligned}
$$

The two-loop anomalous dimensions are,

$$
\begin{aligned}
\gamma_{Q}^{(2)}= & \frac{1}{16 \pi^{2}}\left[-2 Y_{Q 1}\left(\gamma_{\phi_{1}}^{(1)}+\gamma_{Q^{c}}^{(1)}\right)-2 Y_{Q 2}\left(\gamma_{\phi_{2}}^{(1)}+\gamma_{Q^{c}}^{(1)}\right)\right. \\
& \left.-\left(\frac{8}{3} \alpha_{c}+\frac{3}{2} \alpha_{L}+\frac{1}{12} \alpha_{B-L}\right) \gamma_{Q}^{(1)}+\frac{8}{3} b_{3} \alpha_{3}^{2}+\frac{3}{2} b_{L} \alpha_{L}^{2}+\frac{1}{12} b_{B-L} \alpha_{B-L}^{2}\right], \\
\gamma_{Q^{c}}^{(2)}= & \frac{1}{16 \pi^{2}}\left[-2 Y_{Q 1}\left(\gamma_{\phi_{1}}^{(1)}+\gamma_{Q}^{(1)}\right)-2 Y_{Q 2}\left(\gamma_{\phi_{2}}^{(1)}+\gamma_{Q}^{(1)}\right)\right. \\
& \left.-\left(\frac{8}{3} \alpha_{c}+\frac{3}{2} \alpha_{R}+\frac{1}{12} \alpha_{B-L}\right) \gamma_{Q^{c}}^{(1)}+\frac{8}{3} b_{3} \alpha_{3}^{2}+\frac{3}{2} b_{R} \alpha_{R}^{2}+\frac{1}{12} b_{B-L} \alpha_{B-L}^{2}\right], \\
\gamma_{L}^{(2)=} & \frac{1}{16 \pi^{2}}\left[-2 Y_{L 1}\left(\gamma_{\phi_{1}}^{(1)}+\gamma_{L^{c}}^{(1)}\right)-2 Y_{L 2}\left(\gamma_{\phi_{2}}^{(1)}+\gamma_{L^{c}}^{(1)}\right)\right. \\
& \left.-\left(\frac{3}{2} \alpha_{L}+\frac{3}{4} \alpha_{B-L}\right) \gamma_{L}^{(1)}+\frac{3}{2} b_{L} \alpha_{L}^{2}+\frac{3}{4} b_{B-L} \alpha_{B-L}^{2}\right] \\
\gamma_{L^{c}}^{(2)}= & \frac{1}{16 \pi^{2}}\left[-2 Y_{L 1}\left(\gamma_{\phi_{1}}^{(1)}+\gamma_{L}^{(1)}\right)-2 Y_{L 2}\left(\gamma_{\phi_{2}}^{(1)}+\gamma_{L}^{(1)}\right)\right.
\end{aligned}
$$




$$
\begin{aligned}
& \left.-\left(\frac{3}{2} \alpha_{R}+\frac{3}{4} \alpha_{B-L}\right) \gamma_{L^{c}}^{(1)}+\frac{3}{2} b_{R} \alpha_{R}^{2}+\frac{3}{4} b_{B-L} \alpha_{B-L}^{2}\right] \\
\gamma_{\phi_{i}}^{(2)}= & \frac{1}{16 \pi^{2}}\left[-Y_{L i}\left(\gamma_{L}^{(1)}+\gamma_{L^{c}}^{(1)}\right)-3 Y_{Q_{i}}\left(\gamma_{Q}^{(1)}+\gamma_{Q^{c}}^{(1)}\right)\right. \\
& \left.-\left(\frac{3}{2} \alpha_{L}+\frac{3}{2} \alpha_{R}\right) \gamma_{\phi_{i}}^{(1)}+\frac{3}{2} b_{L} \alpha_{L}^{2}+\frac{3}{2} b_{R} \alpha_{R}^{2}\right] .
\end{aligned}
$$

\subsection{Beta function coefficients for the gauge couplings}

The two loop RGE for the gauge couplings are given in general by Eqn (6). The coefficients $b_{i}, b_{i j}$ and $a_{i k}$ depend on the scale range considered, and are listed below:

(A) For $M_{Z} \leq \mu \leq M_{I}$ the coefficients are:

$$
\begin{gathered}
b_{i}=\left(\begin{array}{c}
2 n_{f}+\frac{3}{5} n_{d} \\
-6+2 n_{f}+n_{d} \\
-9+2 n_{f}
\end{array}\right) \quad i=1,2,3 . \\
b_{i j}=\left(\begin{array}{ccc}
\frac{38}{15} n_{f}+\frac{9}{25} n_{d} & \frac{6}{5} n_{f}+\frac{9}{5} n_{d} & \frac{88}{15} n_{f} \\
\frac{2}{5} n_{f}+\frac{3}{5} n_{d} & -24+14 n_{f}+7 n_{d} & 8 n_{f} \\
\frac{11}{5} n_{f} & 3 n_{f} & -54+\frac{68}{3} n_{f}
\end{array}\right) \quad i, j=1,2,3 . \\
a_{i k}=\left(\begin{array}{ccc}
\frac{26}{5} & \frac{14}{5} & \frac{18}{5} \\
6 & 6 & 2 \\
4 & 4 & 0
\end{array}\right) \quad i=1,2,3, k=t, b, \tau .
\end{gathered}
$$

(B) For $M_{I} \leq \mu \leq M_{U}$ : 


$$
\begin{aligned}
& b_{i}=\left(\begin{array}{c}
2 n_{f}+\frac{3}{2}\left(n_{L}+n_{R}\right) \\
-6+2 n_{f}+n_{H}+n_{L} \\
-6+2 n_{f}+n_{H}+n_{R} \\
-9+2 n_{f}+3 n_{c}
\end{array}\right) \quad i=B-L, L, R, 3 \\
& b_{i j}=\left(\begin{array}{cccc}
\frac{7}{3} n_{f}+\frac{9}{4}\left(n_{L}+n_{R}\right) & 3 n_{f}+\frac{9}{2} n_{L} & 3 n_{f}+\frac{9}{2} n_{R} & \frac{8}{3} n_{f} \\
n_{f}+\frac{3}{2} n_{L} & -24+14 n_{f}+7\left(n_{H}+n_{L}\right) & 3 n_{H} & 8 n_{f} \\
n_{f}+\frac{3}{2} n_{R} & 3 n_{H} & -24+14 n_{f}+7\left(n_{H}+n_{R}\right) & 8 n_{f} \\
\frac{1}{3} n_{f} & 3 n_{f} & 3 n_{f} & -54+\frac{68}{3} n_{f}+34 n_{c}
\end{array}\right) \\
& i, j=B-L, L, R, 3 . \\
& a_{i k}=\left(\begin{array}{cccc}
2 & 2 & 6 & 6 \\
12 & 12 & 4 & 4 \\
12 & 12 & 4 & 4 \\
8 & 8 & 0 & 0
\end{array}\right) \quad i=B-L, L, R, 3, k=Q 1, Q 2, L 1, L 2
\end{aligned}
$$

\subsection{Proton decay parameters}

The proton decay formula is given by Eqn (9) [24]: where we have assumed the following values for the parameters [33:

$$
\begin{gathered}
D=0.81 ; F=0.44 ; f_{\pi}=139 \mathrm{MeV} \\
m_{N}=0.938 \mathrm{GeV} ;\left|V_{u d}\right|=0.975 ; \alpha=0.03 \mathrm{GeV}^{3} .
\end{gathered}
$$

The value of $\alpha$ is taken in such a way that proton decay is maximal. In fact, its value has a range from 0.03 to $0.003 \mathrm{GeV}^{3}$. The value of the renormalization factor $A_{R}$ varies from 2.7 to 4.1 The values are plotted in as can be seen in Fig. (4). 


\subsection{The range of $\eta_{b}$}

Here we give the values of $\eta_{b}$ [26] used to extrapolate $m_{b}\left(m_{t}\right)$ to $m_{b}\left(m_{b}\right)$, we have calculated its variation with $\alpha_{3}\left(M_{Z}\right)$ for the different models studied in the text in Fig. (5). We have taken $\alpha_{2}\left(M_{Z}\right)=0.3371$ and $\alpha_{1 Y}\left(M_{Z}\right)=0.01696$. Our calculation agrees with that of Ref. [26] quite well. 


\section{References}

[1] Y. Fugimoto, Phys. Lett 89B, 347 (1980); S. Rajpoot, Phys. Rev. D22, 2244 (1980); F. del Aguila and L. E. Ibáñez, Nucl. Phys. B177, 60 (1981); R. N. Mohapatra and G. Senjanović, Phys. Rev. D 27, 1601 (1983).

[2] J. C. Pati and A. Salam, Phys. Rev D 10, 275 (1974). R. N. Mohapatra and J. C. Pati, Phys. Rev. D11, 566 (1975). G. Senjanović and R. N. Mohapatra, Phys. Rev. D 12, 1502 (1975).

[3] Such a scale is desirable if $\nu_{\tau}$ has to constitute the Hot Dark Matter of the universe. See for example, R. Shafer and Q. Shafi, Nature, 359, 199 (1992).

[4] M. Gell-Mann, P. Rammond and R. Slansky, in Supergravity, eds D. Freedman et al, North Holland Publishing Company, Amsterdam, 1980; T. Yanagida, in Proceedings of the KEK workshop, 1979 (unpublished). R. N. Mohapatra and G. Senjanović. Phys. Rev. Lett 44, 912 (1980).

[5] J. E. Kim, Phys. Rep. 150, 1 (1987) and references there in; R. D. Peccei, in CP violation, ed. C. Jarlskog, (World Scientific), 1989.

[6] M. Bando, J. Sato and T. Takahashi, Phys.Rev. D52, 3076 (1995).

[7] N.G. Deshpande, E. Keith, T.G. Rizzo, Phys.Rev.Lett. 70, 3189 (1993).

[8] D. G. Lee and R. N. Mohapatra, Phys.Rev. D52, 4125 (1995).

[9] B. Brahmachari and R. N. Mohapatra, Phys. Lett. B 357566 (1995). 
[10] F. Vissani and A. Yu. Smirnov, Phys. Lett 341B, 173 (1994).

[11] A. Brignole, H. Murayama and R. Rattazi, Phys. Lett. B335, 345 (1994).

[12] F. Abe et. al., Phys. Rev. D50, 2966 (1994). Particle Data Group, Physical Review D50, 1173 (1994).

[13] Review of Particle Properties, Particle Data Group, Phys. Rev. D52 (1994).

[14] S. Bethke, International Conference on High Energy Physics (ICHEP 92), 26th, Dallas, 6-12 Aug 1992. HIGH ENERGY PHYSICS, proceedings. Edited by James R. Sanford. American Inst. Phys., 1993. 2v. (AIP Conference Proceedings, 272).

[15] M. Kobel, DESY report, DESY F31-91-03; Z. Phys. C53, 193 (1992).

[16] A. X. El-Kadra, G. Hockney, A. Kornfeld and P. Mackenzie, Phys. Rev. Lett 69, 729 (1992). C. Davies, K. Hornbostel, G. Lepage, A. Lidsey, J. Shigemitsu and J. Sloan, Phys. Lett. 345B, $42(1995)$.

[17] M. Virchaux and A. Milsztajn, Phys. Lett. 274B, 221 (1992).

[18] B. Brahmachari and R. N. Mohapatra, IC-95-217, hep-ph/9508293.

[19] S. Choudhuri, S. Chung and J. Lykken, Nucl.Phys. B456, 89 (1995); Talk given at Particles, Strings, and Cosmology (PASCOS 94), Syracuse, NY, 19-24 May 1994. In *Syracuse 1994, Proceedings, PASCOS '94*223-233, and Ohio State U. Columbus - OHSTPY-HEP-T-94-007 (94/08,rec.Oct.); G. Aldazabal, A. Font, L. Ibáñez and A. M. Uranga, Nucl.Phys. B452, 3 (1995). 
[20] M. Masip and A. Rasin, Phys.Rev. D52, 3768 (1995); UMD-PP-96-17, hep-ph/9508365.

[21] J. Bagger, K. Matchev and D. Pierce, Phys. Lett. B 348, 443 (1995); P. H. Chankowski, Z. Pluciennik and S. Pokorski, Nucl. Phys. B 439, 23 (1995); M. Bastero-Gil and J. PérezMercader, Nucl Phys. B450, 21 (1995).

[22] K. S. Babu and S. M. Barr, Phys. Rev. D 48, 5354 (1993); ibid, D 512467 (1995).

[23] R. Tarrach, Nucl. Phys. B183, 384 (1981)

[24] J. Hisano, H. Murayama and T. Yanagida, Phys. Rev. Lett. 69, 1014 (1992); Nucl. Phys. B 402, 46 (1993).

[25] L. E. Ibáñez and C. Muñoz, Nucl. Phys. B295, 425 (1984), and L. E. Ibáñez and F. J. Yndurain, Phys. Lett. 113B, 367 (1984).

[26] V. Barger, M. S. Berger and P. Ohmann, Phys. Rev. D 47, 1093 (1993).

[27] C. T. Hill, Phys.Rev. D24 (1981); B. Pendleton, G.G. Ross, Phys.Lett. 98B, 291 (1981).

[28] R. N. Mohapatra, Phys. Rev. Lett, 56, 561 (1986). R. N. Mohapatra and J. W. F. Valle, Phys. Rev. D34, 1642 (1986).

[29] S.A. Bludman, D.C. Kennedy and P.G. Langacker, Nucl. Phys. B374, 373 (1992); Phys.Rev. D45, 1810 (1992).

[30] K.S. Babu, Phys.Lett. B319, 191 (1993).

[31] M. K. Parida and M. Rani, North-Eastern Hill University (India), REPORT (1996). 
[32] K. Inoue et al, Prog. Theor. Phys. 68, 927, (1982); ibid 67, 1889 (1982). J. E. Bjorkman and D. R. T. Jones, Nucl. Phys. B259, 533 (1985).

[33] M. Cloudson, M. B. Wise and L. Hall, Nucl. Phys. B82, 297 (1982); S. Chada and M. Dauiels, Nucl. Phys. B229, 105 (1983). 

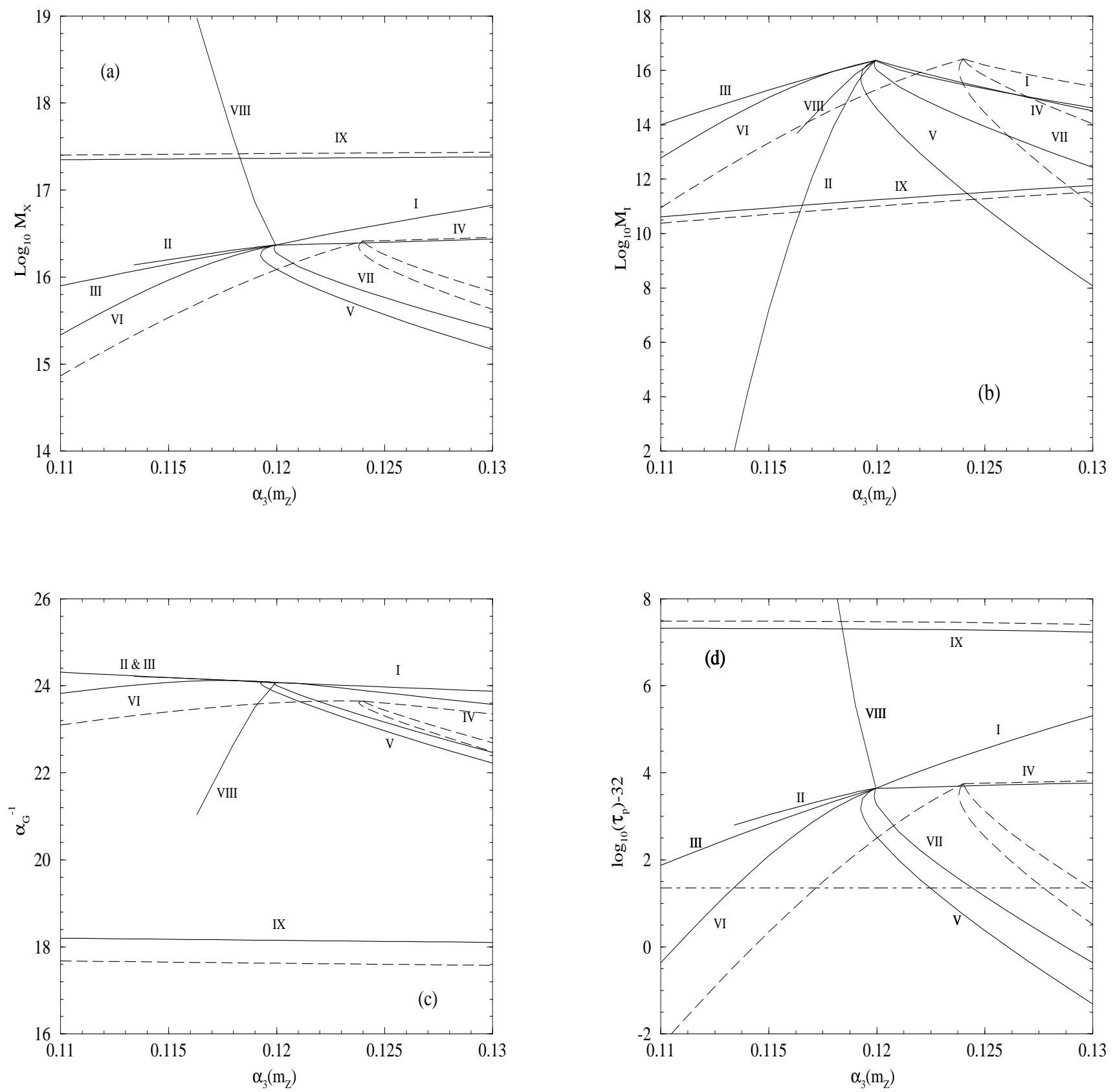

Figure 1: Predictions for (a) Unification scale, (b) Intermediate scale, (c) Unification gauge coupling, and (d) Proton life-time, for the models listed in Table I. Solid lines denote high $\tan \beta$ $\left(Y_{1}\left(M_{X}\right)=Y_{2}\left(M_{X}\right)=1\right.$; dashed lines denote the low $\tan \beta$ regime $\left(Y_{1}\left(M_{X}\right)=1, Y_{2}\left(M_{X}\right)=10^{-4}\right)$. In Figure (d) the dotted line is the experimental limit $\tau_{p}=5.5 \times 10^{32} \mathrm{yr}$. 

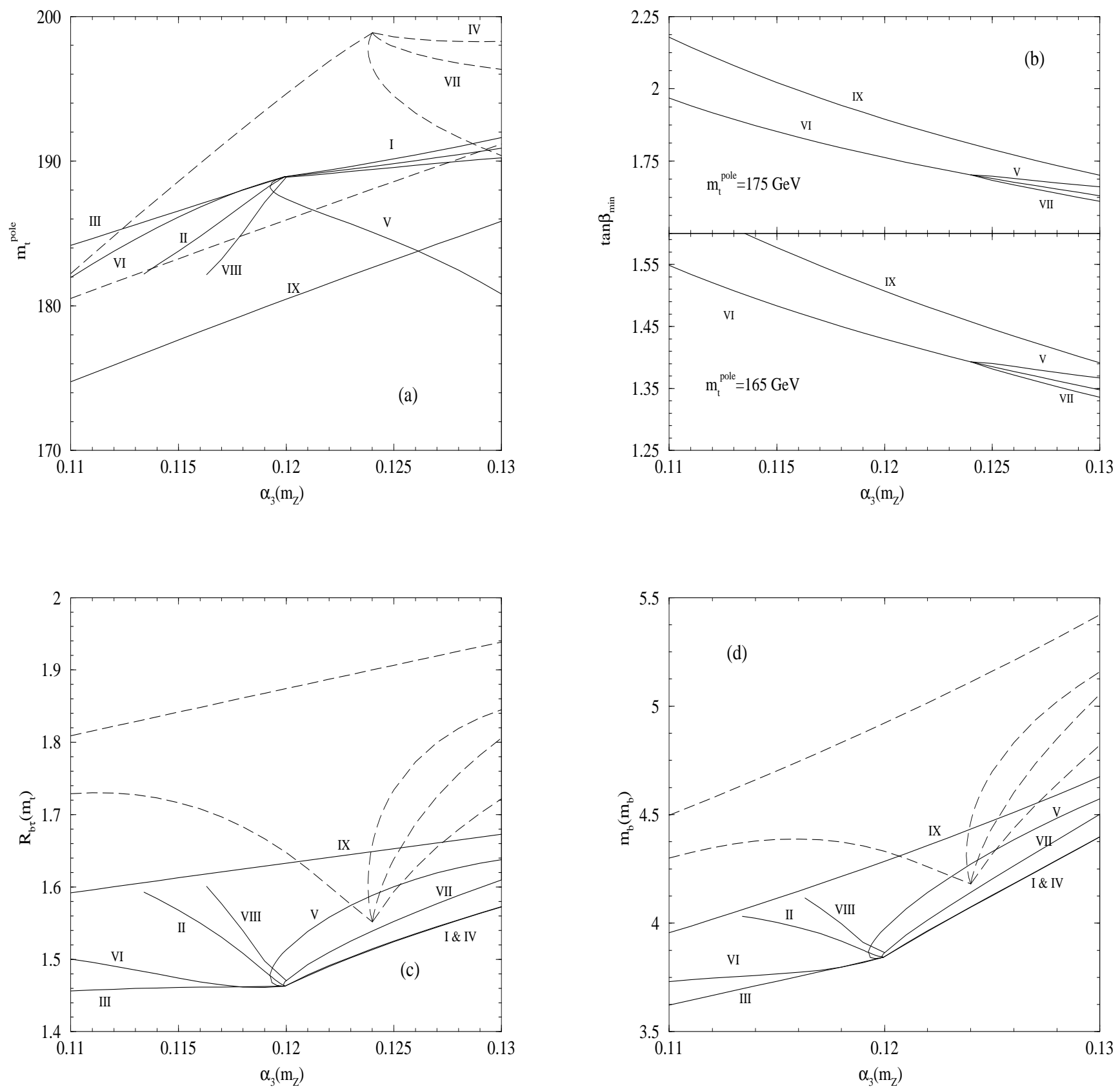

Figure 2: Predictions of (a) pole mass of the top quark, (b) lower bound on $\tan \beta$, (c) $R_{b \tau}$ at $m_{t}$, (d) running mass of the $b$ quark. Solid lines and dashed lines are as in Fig. (1). 

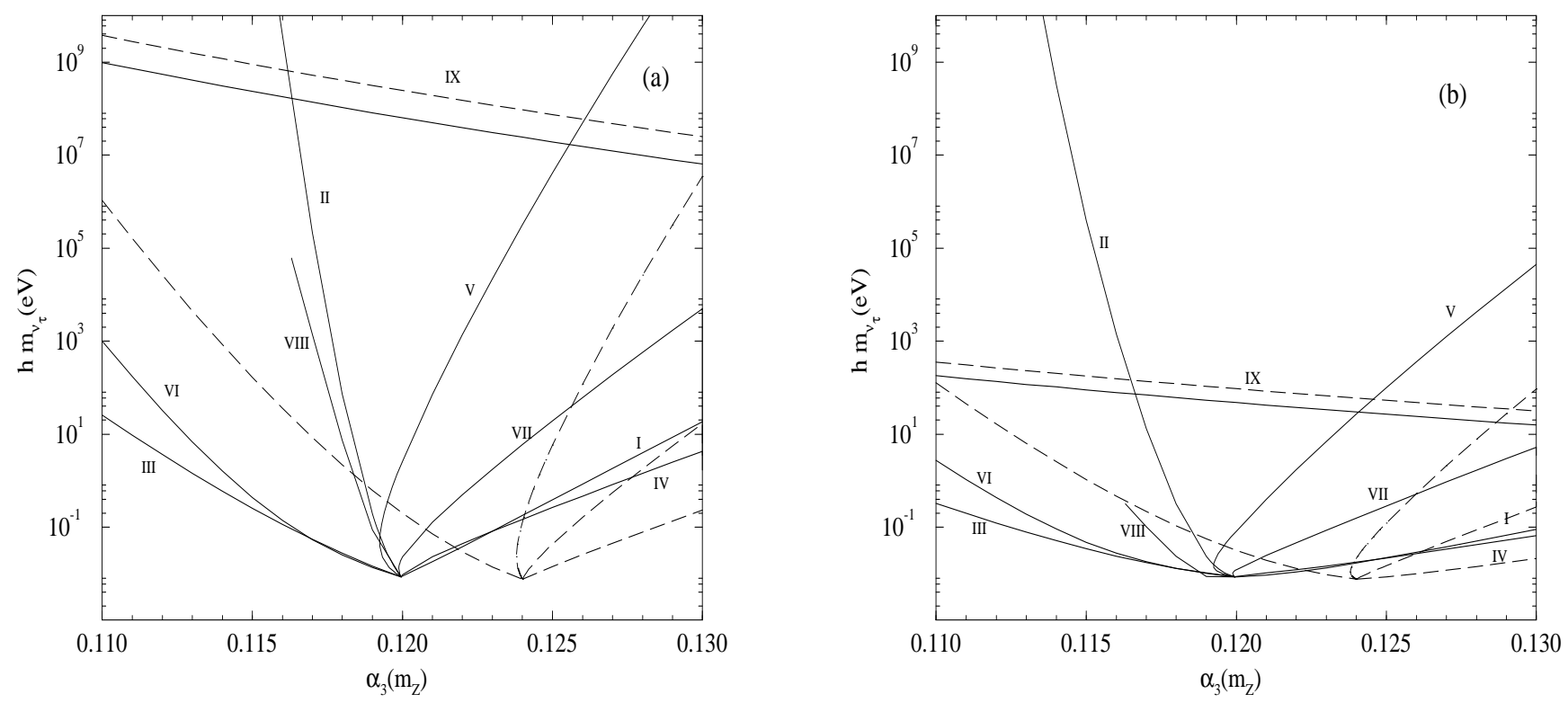

Figure 3: Predictions of the left-handed neutrino mass by see-saw mechanism by the two scenarios (a) and (b). Solid and dashed lines are as before.

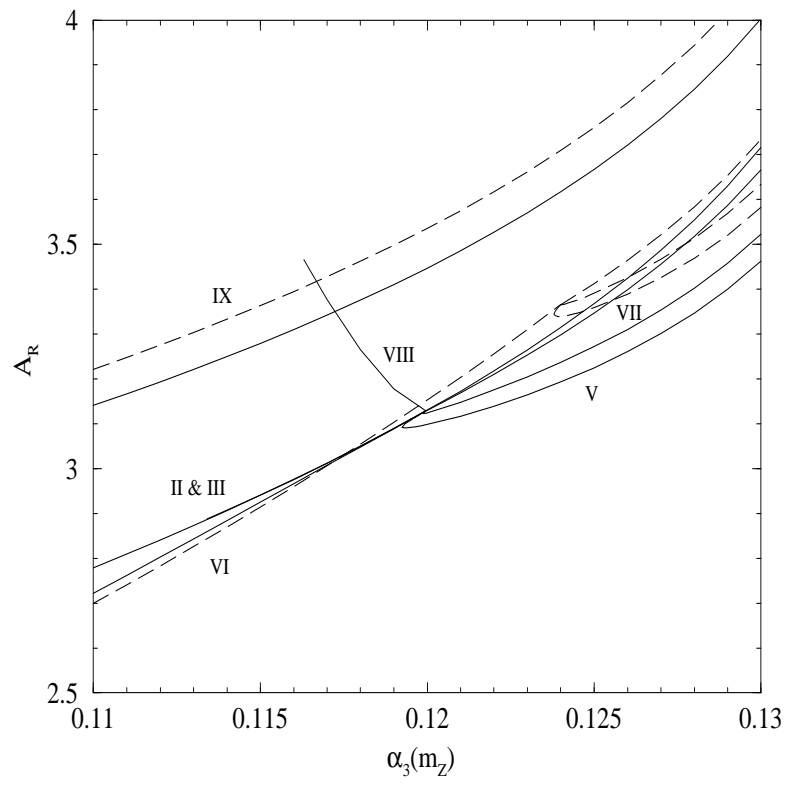

Figure 4: Variation of $A_{R}$ with respect to $\alpha_{3}\left(M_{Z}\right)$ in different models. Solid and dashed lines are as before. 


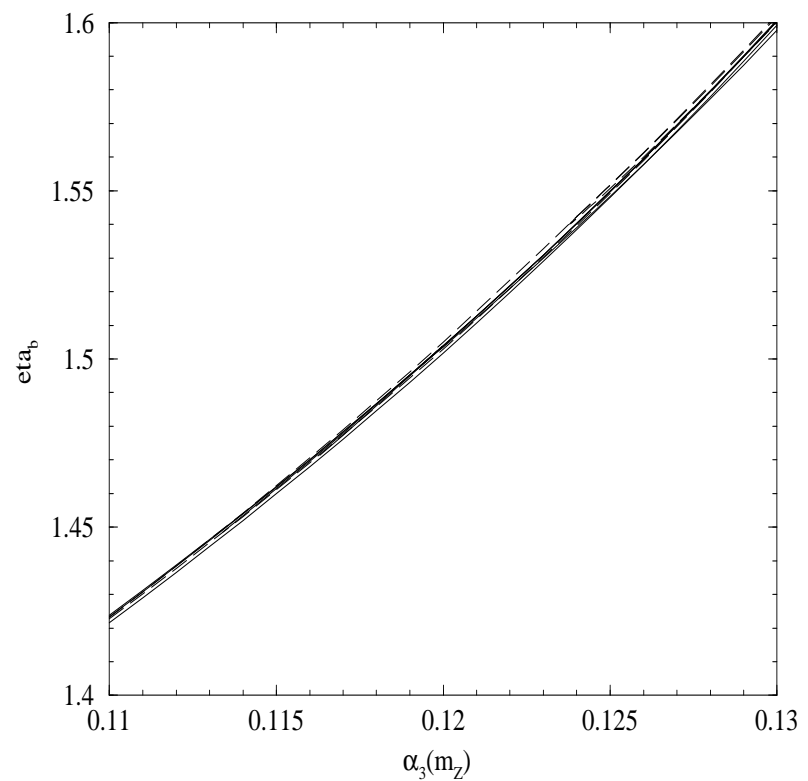

Figure 5: Variation of $\eta_{b}$ with respect to $\alpha_{3}\left(M_{Z}\right)$ in different models. Solid and dashed lines are as before. 